Z Rheumatol 2009 $68: 527-528$

DOI 10.1007/s00393-009-0504-9

๑) Springer Medizin Verlag 2009
B. Manger ${ }^{1} \cdot$ E. Märker-Hermann ${ }^{2}$

${ }^{1}$ Medizinische Klinik III mit Poliklinik, Universität Erlangen-Nürnberg, Erlangen

${ }^{2}$ Klinik Innere Medizin IV (Rheumatologie, klinische Immunologie und

Nephrologie), HSK Dr. Horst Schmidt Kliniken GmbH, Wiesbaden

\title{
Rheuma Update 2009
}

\section{Ausgewählte Manuskripte jetzt im Online-Bereich der Zeitschrift für Rheumatologie verfügbar}

Liebe Kolleginnen und Kollegen,

vor viereinhalb Jahren haben wir mit einem kleinen Team von Kollegen und in Kooperation mit der Rheumaakademie das Rheuma Update aus der Taufe gehoben. Mit dieser Veranstaltung betraten wir Neuland im Bereich der rheumatologischen Fortbildung, denn das Konzept unterscheidet sich in wichtigen Punkten von bisherigen Formaten:

Im Mittelpunkt der Vorträge und Manuskripte stehen die Erkenntnisse aus Arbeiten, die im jeweils vergangenen Jahr in „Peer-Review-Journals“ oder in wichtigen Ausnahmen auch als Abstracts publiziert wurden - ein echtes Update also, das in zwei Tagen einen konzentrierten Überblick über die aktuellsten Entwicklungen im gesamten Spektrum rheumatologischer Themen gibt. Die Ergebnisse der Publikationen bringen wir dabei auf den für Ihre ärztliche Tätigkeit wichtigsten Punkt: die Konsequenzen für Diagnostik und Therapie in Klinik und Praxis. Der ausführlichen Diskussion der präsentierten Daten und Schlussfolgerungen bieten wir dabei großzügigen Raum - in kurzen Diskussionsrunden während und am Ende des Vortrags, im anschließenden „Speaker’s Corner" und während der ganzen Veranstaltung. Deshalb ist das komplette Team an beiden Tagen vor Ort und steht für Rückfragen und weitere Diskussionen bereit.

Wir waren von Anfang an überzeugt, dass unsere Veranstaltung mit diesem da- mals neuen Update-Konzept eine wichtige Ergänzung im bis dahin vorhandenen Angebot an rheumatologischen Fortbildungen darstellt. Und wir hofften selbstverständlich, dass auch Sie - als Rheumatologen oder rheumatologisch interessierte Ärzte - das Besondere an diesem Seminar sehen und teilnehmen würden. Der tatsächliche Zuspruch übertraf aber dann doch alle Erwartungen.

\section{(Die aktuellsten Entwicklungen und ihre Umsetzung in der Praxis stehen im Mittelpunkt}

Schon zur Premiere im Jahr 2006 begrüßten wir mehr als 300 Teilnehmer, und seitdem lockt das Rheuma Update jährlich über 450 Rheumatologen nach Wiesbaden, viele kommen sogar jedes Jahr wieder. Umso erfreulicher wird dieser Erfolg, wenn man bedenkt, dass jeder einzelne Teilnehmer mit seiner Teilnahmegebühr in bedeutendem Maße zur Realisierung des Seminars beiträgt.

Von großer Bedeutung für den Erfolg unseres Rheuma Updates und für die Teilnehmer ein wertvoller Bestandteil ist das umfangreiche Handbuch zum Seminar. Dieses enthält die ausführlichen Manuskripte der Referenten zu allen wichtigen Publikationen des vergangenen Jahres aus ihrem jeweiligen Themenbereich. Nicht an die zeitlichen Vorgaben eines Vortrags gebunden, finden sich in diesen Texten noch ausführlichere Informationen, und sie berücksichtigen zudem weitere, unseren
Referenten wichtig erscheinende Studien aus dem Vorjahr. Selbstverständlich auch hier: kritisch kommentiert und mit einem Fazit für die praktische und klinische Arbeit versehen. Das exklusiv nur für Teilnehmer erhältliche Handbuch dient damit nicht nur zur Vertiefung der gehörten Vorträge, sondern stellt für viele ein unentbehrliches Nachschlagewerk dar.

In Kooperation mit dem SpringerVerlag und speziell der Zeitschrift für Rheumatologie stellen wir für Abonnenten dieser Zeitschrift im Online-Bereich nun erneut drei Manuskripte aus unserem Handbuch zur Verfügung. Es handelt sich um die Beiträge des Rheuma Updates $2009 \mathrm{zu}$ den Teilseminaren „Osteologie und Physikalische Medizin“, „Spondyloarthritiden I“ und „Sjögren, Myositis, progressive systemische Sklerose (PSS)“.

Das Manuskript „Osteologie und Physikalische Medizin“, erstellt von Prof. Uwe Lange, Kerckhoff-Klinik Bad Nauheim, geht im osteologischen Teil u. a. ausführlich auf die Zusammenhänge zwischen Bisphosphonat-Therapie und Frakturrate ein. Prof. Lange wirft einen kritischen Blick auf die Kalziumsupplementation zur Prophylaxe und fasst sehr informativ innovative Therapieverfahren zusammen. Der zweite Manuskriptteil geht verstärkt auf die evidenzbasierte physikalische Therapie bei Osteoporose und in der Rheumatologie ein und widerlegt den Vorwurf, die Wirksamkeit dieser Therapie zeige sich nur in empirischen Daten. 
In seinem Beitrag "Spondyloarthritiden I" widmet sich Dr. Martin Rudwaleit von der Berliner Charité bei der Betrachtung klinischer Aspekte ausführlich dem Zusammenhang zwischen akuter Entzündung, Anti-TNF-Therapie und Röntgenprogression bei ankylosierender Spondylitis. Im Abschnitt zur Therapie dominieren ebenfalls die neuesten Erkenntnisse zur TNF-Blocker-Therapie mit Biologika, aber auch aktuelle Daten zur Physiotherapie und NSAR finden sich hier.

Sowohl für das Sjögren-Syndrom als auch für die Myositis und die PSS stehen aktuelle Studien zur Therapie im Mittelpunkt des Manuskripts von Frau Dr. Gabriela Riemekasten, ebenfalls von der Charité in Berlin. Im Rahmen des PSS-Kapitels finden sich aber auch aktuelle und spannende Daten zur Erfassung, Pathogenese und Therapie der pulmonalen Hypertonie.

Wir denken, dass diese auch jetzt noch hoch aktuellen Beiträge von profundem Interesse für Sie sind, und hoffen, dass Sie und Ihre Klinik oder Praxis von diesen Arbeiten profitieren. Sollten wir Ihr Interesse für unsere Veranstaltung, das Rheuma Update, wecken, würden wir uns selbstverständlich freuen, Sie 2010 dort begrüßen zu können. Unser kleines Jubiläum die 5. Veranstaltung - findet am 12. und 13. März 2010 am gewohnten Ort in Wiesbaden statt. Dort bieten wir Ihnen wieder einen konzentrierten Überblick über die für Rheumatologen wichtigsten Studie aus dem Jahr 2009 und versprechen Ihnen eine optimale Fortbildung, um sich in der Rheumatologie und ihren Grenzgebieten auf den neuesten Stand des Wissens $\mathrm{zu}$ bringen.

\section{Mit kollegialen Grüßen}

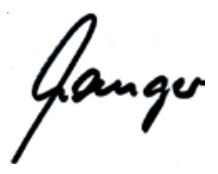

B. Manger<smiles>CC(C)CC(C)C</smiles>

E. Märker-Hermann

\section{Korrespondenzadresse}

Prof. Dr. E. Märker-Hermann

Klinik Innere Medizin IV (Rheumatologie,

klinische Immunologie und Nephrologie), HSK Dr. Horst Schmidt Kliniken GmbH

Ludwig Erhard Str. 100, 65199 Wiesbaden

elisabeth.maerker-hermann@hsk-wiesbaden.de

\section{Zusatzmaterial online ...}

Die Handbuchbeiträge des Rheuma Updates 2009 finden Sie online im Volltextarchiv der Zeitschrift für Rheumatologie im Anhang dieses Editorials. Bitte folgen Sie dem Pfad: www.ZeitschriftfuerRheumatologie.de $>$ Online-Version > Beitrag > Electronic Supplementary Material oder geben Sie folgende URL ein: dx.doi.org/10.1007/s00393-009-0504-9.

\section{Kaffeekonsum fördert die Gesundheit}

Im Durchschnitt trinkt jeder deutsche Bundesbürger 148 Liter Kaffee im Jahr. Das Institut für Prävention und Ernährung in München beschäftigt sich mit den Auswirkungen des regelmäßigen Genusses auf die Gesundheit und welches Krankheitsrisiko damit verbunden ist. In einer Übersichtsarbeit wurden nun zahlreiche Studien aus den vergangenen zehn Jahren ausgewertet. Die Schwerpunkte lagen hierbei auf kardiovaskuläre Krankheiten, Typ-2-Diabetes, Tumorerkrankungen sowie den Einfluss von Kaffee auf die kognitiven Fähigkeiten und die allgemeine Mortalität. Die aktuellen Studiendaten weisen darauf hin, dass der Genuss von Kaffee bestimmte Krankheitsrisiken deutlich vermindern kann und daher eine präventionsmedizinische Relevanz besitzt. Eine Reduzierung des Kaffeekonsums als allgemeine Gesundheitsempfehlung erscheint aufgrund der neuesten wissenschaftlichen Erkenntnisse nicht notwendig.

Literatur: Vormann J (2009) Ärzte-Merkblatt "Präventionsmedizinische Aspekte des Kaffeekonsums"

Quelle:

Deutsches Grünes Kreuz e.V. (DGK), www.kaffee-wirkungen.de 